\title{
Pharmacology and Statistics: Recommendations to Strengthen a Productive Partnership
}

\author{
Reynold Spector ${ }^{a, b} \quad$ Elliot S. Vesell ${ }^{c}$ \\ ${ }^{a}$ Harvard, MIT Program in the Health Sciences, Cambridge, Mass., ${ }^{b}$ Robert Wood Johnson Medical School, \\ New Brunswick, N.J., and ' Department of Pharmacology, Pennsylvania State University College of Medicine, \\ Hershey, Pa., USA
}

\section{Key Words}

Statistics in pharmacology $\cdot$ Recommendations $\cdot$ Bias · Confounding $\cdot$ Dropout

\begin{abstract}
Critical to the discovery, development and rational use of drugs and vaccines are the foundational principles and proper application of statistics. However, in too many cases, there has been misuse of statistics and/or overemphasis on statistical significance $(p<0.05)$, as though this criterion possessed truth-guaranteeing properties. To clarify confusion about the proper use of statistics in pharmacology, we summarize briefly the foundational principles of probability; the role of statistics in assessment of causality; the three basic uses of statistical methods, especially those employed in hypothesis testing; and current statistical issues in pharmacological research. We then review and provide examples of the meaning of statistical significance, the consequences of lack of randomization in epidemiology/observation studies, the criteria for measurement instrument validation, the problems with subgroup analyses, the need for multiple comparison statistical methods, and how to handle dropouts and missing data. Finally, based on sound experimental and statistical principles, we make a series of recommendations to both experimentalists and journal editors to improve published pharmacological experiments. These include widespread use of blinding and randomization and/or random selection of subjects in both basic and clinical phar-
\end{abstract}

macology, mandatory use of rigorous evidentiary criteria in epidemiology/observation studies claiming causal associations, proper interpretation of statistical versus clinical/ pharmacological significance, appropriate interpretation of meta-analyses, meaningful validation of methods, and a more rational statistical approach to subgroup analyses and genetic association studies. Copyright $\odot 2006 \mathrm{~S}$. Karger AG, Basel

\begin{abstract}
No formal statistical tests can answer those questions (causality).

Bradford Hill (1965) in his discussion of potential conclusions derived from epidemiology/observation studies
\end{abstract}

\section{Introduction}

Brilliant philosophers, pharmacologists and statisticians have developed sound foundational principles and practical scientific methods to study drugs and vaccines [1-6]. Much attention focused on discovering cause and effect relationships [5-8]. Since ultimate goals of pharmacology (encompassing both basic and clinical pharmacology) are prevention and treatment of disease and illness, pharmacological experiments must be performed, interpreted and reported correctly.

The scientific methods and principles described in this commentary are productive $[1,7-9]$. They have been

\section{KARGER \\ Fax +4161306 1234 E-Mail karger@karger.ch} www.karger.com (c) 2006 S. Karger AG, Basel 0031-7012/06/0783-0113\$23.50/0

Accessible online at:

www.karger.com/pha
Elliot S. Vesell, MD

Department of Pharmacology, Pennsylvania State University College of Medicine 500 University Drive

Hershey, PA 17033 (USA)

Tel. +1 717531 8285, Fax +1 717531 5013,E-Mail esvl@psu.edu 
Table 1. Key methodological components for testing the null hypothesis in clinical trials $[1,4,5,14,15,39]$

1 Single hypothesis (e.g., treatment = placebo)

2 Single variable (treatment) and single outcome measure

3 Proper selection and randomization of an adequate number of subjects

4 Blinding where appropriate

5 Documentation of compliance

6 Complete follow-up of all subjects

7 Proper statistical analysis

8 Conclusion

9 Generalization as appropriate (see text)

Often, the objective of trialists is to falsify the null hypothesis so the treatment can be concluded to be 'better' than placebo (or another treatment) on the outcome variable or, in other words, that the treatment 'causes' improved health, at least as far as the primary outcome variable is concerned.

conscientiously and successfully employed by experienced investigators [7-9]. Moreover, they are recognized as the 'gold standard' by the FDA and European regulatory authorities $[7,8]$. An example of these methods, shown in table 1 , tests the null hypothesis that a clinical treatment is not superior to a placebo $[1,4,7]$.

Nevertheless, many articles, even in 'high quality' journals, report pharmacological observations and conclusions claiming validity solely on statistical significance of the data $(\mathrm{p}<0.05)[8,10,11]$. Clinical recommendations are often based on such work, as though $\mathrm{p}<$ 0.05 possessed truth-guaranteeing properties. The results, however, are often later shown to be misleading, inconsequential or even incorrect $[8,10]$. Unfortunately, news media frequently disseminate such work uncritically before careful evaluation and replication can validate it.

Why does this happen, when there should be clear understanding of proper pharmacological and statistical methodology, especially by journal editors and sophisticated news organizations? Why are sound principles so often breached? Why did Bradford Hill in the above quotation claim that formal statistical tests in certain types of studies cannot answer causal questions?

Before addressing these issues, we briefly review the notions of causality, including our perspective on the problem of induction (Hume) and its modern 'solution'. Then, we discuss philosophical and scientific bases of statistics and probability, especially principles relevant to pharmacology. With this background we will provide key definitions, making explicit important assumptions and methods. Next, we evaluate several basic and clinical studies describing the strengths and weaknesses of specific experimental and statistical procedures and methods. Once foundations and principles of proper experimental design and statistics are understood, evaluation of pharmacological studies is facilitated. In the final section, we offer a series of recommendations on how to improve certain pharmacological experiments and publications.

\section{Background}

In recent publications, we and others have discussed truth, underlying assumptions in medical science, various notions of causality, general evidential criteria for establishing causality in the pharmacological sciences, and myriad challenges to these current principles, including Quine's correct notions of holism and underdetermination of theory with data $[1,4,5,7,10]$. We described how these challenges have been overcome, topics relevant to the current discussion [7]. We also documented the beneficent consequences of the successful application of modern scientific pharmacology to drug discovery and development $[1,9]$.

To focus on another critical aspect of the intersection of pharmacology, probability and statistics, we return to Hume, who recognized that our notion of causality rests on the association of events $A$ and $B[7,12]$. When event A consistently preceded event B, it was often labeled, sometimes incorrectly, the cause of B. Subsequent philosophers have refined and extended Hume's thinking about scientific causality into the two most common categories: (1) necessary and sufficient causality and (2) contributory causality, in which myriad causal or empowering factors exist $[7,12]$. These contributory causes are sometimes termed precipitating, predisposing or sustaining causes. The concept of contributory causality requires statistical thinking and its attendant science since, for example, in treatment response, not everyone who receives a certain 'efficacious' treatment responds, nor does everyone not receiving the treatment fail to respond $[7$, 12]. Much of causal thinking in pharmacology depends on the notion of contributory causality $[2,5-7,12]$. Therefore, because of the nature of contributory causality, large randomized long-term clinical trials, especially when effects are modest or slow, are sometimes required to establish the value of certain treatments, e.g., lowering serum cholesterol with drugs $[1,5,7,8,10]$. 
Table 2. Causality and laws [12]

\begin{tabular}{|c|c|c|c|c|}
\hline & Causality type & Variables & Laws & Examples \\
\hline 1 & $\begin{array}{l}\text { Necessary } \\
\text { and sufficient }\end{array}$ & $\begin{array}{l}\text { one or } \\
\text { few }\end{array}$ & universal & $\begin{array}{l}\text { gravity and planetary } \\
\text { motion }\end{array}$ \\
\hline 2 & Contributory & many & statistical & $\begin{array}{l}\text { reduction of death } \\
\text { on statins, and ulcers on } \\
\text { proton pump inhibitors }\end{array}$ \\
\hline
\end{tabular}

When Carnap [12] reviewed these matters, he noted that the function of science was to describe, explain and predict phenomena. As science progressed, Carnap pointed out how much we have steadily improved our ability not just to classify, but also to compare and quantitate. Carnap also noted that there were fundamentally two types of explanations (laws), depending on the underlying causes: universal laws (often associated with necessary and sufficient causality) which can often be verified precisely and accurately, and statistical laws (table 2). However, Carnap reinforced the notion that both these categories depended on the assumption of regularity in the future, i.e., that the past does, in fact, predict the future. In other words, we must assume that sound science can overcome Hume's 'problem of induction' $[5,7,12]$. Moreover, Carnap conceded that a continuum exists between universal laws and statistical laws [12].

\section{Induction and Statistical Probability}

Over three hundred years ago, mathematicians interested in winning 'games of chance' proposed the 'principle of indifference' to elucidate the 'science' of gaming $[2,12]$. Since so many immeasurable variables participate, the mathematicians assumed over time that none dominates and they average out $[2,12]$. For example, these mathematicians assumed that an unbiased die had a one in six 'chance' of turning up a particular side or number. They developed an elegant and correct statistical methodology based on the principle of indifference (table 3) [2, 3, 12]. However, the principle of indifference does not apply to most medical situations, e.g., response to treatment, mortality, etc. [12]. Therefore, Mises and Reichenbach [see ref. 12] proposed that probability be defined as the relative frequency in not just a finite series of instances, but as the limit of the relative frequency (table 3). This view is a key part of the basis for current medical statistics, when the principle of indifference is not
Table 3. Foundational principles of probability $[2,12,39]$

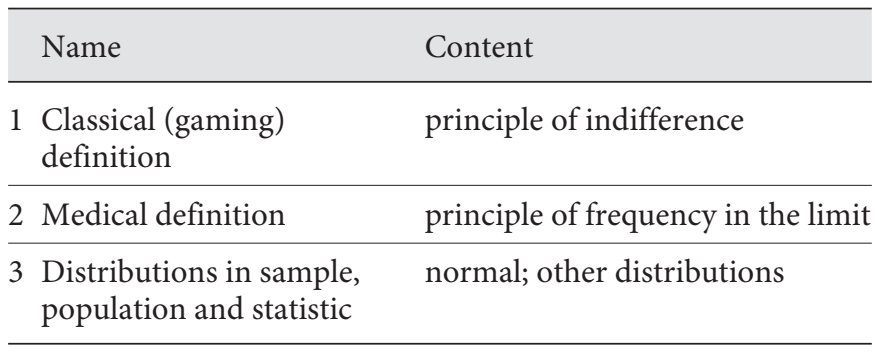

applicable $[2,12]$. For example, take a loaded die. To determine its characteristics, many tosses are required. In the limit, it may be observed that numbers $1,2,3,4,5$, or 6 turn up $25,15,15,15,15$, and $15 \%$ of the time, respectively. With this information, a proper statistical approach can be developed $[2,3,12]$.

Finally, statisticians emphasized that the investigator should, whenever possible, know or experimentally determine the distribution of the sample or population being studied $[2,3,12]$. Is the sample or population normally distributed? When this is not possible, nonparametric ('distribution-free') statistical methods for data evaluation are available and can be applied [3].

In these processes, Carnap [12] stressed the need to separate statistical probability from logical probability (inductive inference). The latter, a separate field, deals with the methods involved in using statistical data (e.g., frequencies or statistical conclusions from trials) and making generalizations or drawing conclusions [12].

\section{Statistical Definitions, Assumptions and Methods}

There are three veridical roles for statistical methods in medical science: estimation, hypothesis testing, and prediction/decision making (table 4) [2, 5, 8]. All statistical methods depend on random, independent sampling procedures, and/or, where appropriate, randomization of subjects (table 4) $[2,3,5,8,12]$. When used for purposes other than those in table 4 , or when the assumptions are not met, statistical arguments can seriously mislead. Before focusing on hypothesis testing, we further define and comment on some terms that create confusion (table 5). In comparative hypothesis testing, randomization is applied (Definition 1c) to a sample population under study to minimize bias and confounding (table 5). A striking example of confounding occurred in the discovery of adrenal gland function and its role in Addison's disease, now known to be due to cortisol/aldosterone de- 
Table 4. Uses of statistical methods [2, 3, 12, 14, 15]

\begin{tabular}{ll}
\hline Type & Examples \\
\hline 1 Estimation & $\begin{array}{l}\text { distribution of height in a } \\
\text { population or longevity }\end{array}$ \\
\hline 2 Hypothesis testing & $\begin{array}{l}\text { comparison of treatments A } \\
\text { versus B (e.g., null hypothesis) }\end{array}$ \\
\hline $\begin{array}{l}\text { Prediction (based on } \\
\text { logical probability/statistical } \\
\text { inference) }\end{array}$ & $\begin{array}{l}\text { how long a diseased } \\
\text { individual will survive on a } \\
\text { certain treatment }\end{array}$ \\
\hline
\end{tabular}

All these methods depend on observations being drawn randomly and independently and/or proper randomization (see table 5 and discussion).

ficiency [2]. Since the adrenal gland was then recognized to secrete epinephrine, the hypothesis was formulated that epinephrine would be helpful in the treatment of Addison's disease. Consequently, epinephrine was given in saline enemas because intravenously it was unsafe. The patients improved, but in retrospect, it was due to the saline not the epinephrine [2]. If the investigators had used a saline control (randomized), they would not have made this 'confounding' error.

The notion of falsification (table 5) also requires comment. Popper [13] and others [7, 11] noted how difficult it is to verify certain propositions (e.g., all swans are white) as Hume emphasized centuries ago, since not every swan can be assessed. Only one black swan will disprove the proposition. Verification is especially difficult in testing contributory causality. In all science, Popper argued successfully that falsification is easier to understand and embrace than verification. Therefore, pharmacological research is often oriented toward falsifying the null hypothesis. The FDA and other regulatory bodies support this approach [1]. However, falsification does not solve the problem of induction. Moreover, in all medical research, subtle undefined and unknown confounding variables (table 5) may still exist.

There are three general methods to test hypotheses (table 6) $[2,3,12]$. The method of Bayes depends on knowledge of prior probability. This is often unknown. Although Bayes' method is very powerful in certain situations, especially when prior probability can be accurately estimated, we focus on the updated methods of Fisher and Neyman-Pearson, since Bayes' method is not widely used in pharmacological studies [2]. Fisher's and Neyman-Pearson's methods encourage proper experimental
Table 5. Definitions and descriptions $[2,39]$

1 Random
(a) Random event: satisfaction of principle of indifference
(b) Random sample: one chosen from a carefully defined population with the aid of a formal method to avoid bias and confounding
(c) Randomization: in comparative trials, a formal method to assign subjects (by chance) to one or the other treatment
(d) Random errors: errors that follow the principle of indifference

2 Bias [39]

(a) The systematic tendency of any factors associated with the design, conduct, analysis, and evaluation of the results of a trial to make the estimate of a treatment effect deviate from its true value

3 Confounding

(a) The effect of two or more variables that do not allow a conclusion about either one separately

4 Validity

(a) In logic, an argument is valid if the conclusions follow from the premises

(b) In pharmacological sciences, a method is valid if it measures what it should, is reproducible and responsive to change, e.g., by a treatment

5 Falsification

(a) In hypothesis testing, a standard easier to satisfy than verification (see text)

Table 6. Statistical methods for hypothesis testing [2, 3, 14, 15, 39]

\begin{tabular}{lll}
\hline \multicolumn{2}{c}{ Statistical method } & Characteristic \\
\hline 1 & Bayes & knowledge of prior probability \\
\hline 2 & $\begin{array}{l}\text { Fisher (updated by } \\
\text { Popper and others) }\end{array}$ & knowledge of frequency \\
\hline 3 & Neyman-Pearson & estimation of power
\end{tabular}

design and allow testing of a hypothesis (especially the null hypothesis) in such a way as to avoid type I (falsely rejecting the null hypothesis; Fisher) and type II errors (falsely accepting the null hypothesis; Neyman-Pearson) $[2,5,14,15]$. Neyman-Pearson analyses encourage enough subjects to power experimental trials sufficiently to avoid or minimize type II errors $[2,5,14,15]$. Many excellent texts describe and exemplify these methods $[3,14,15]$. 
Table 7. Statistical issues in pharmacological research

1 What $\mathrm{p}$ value is statistically significant?

2 Consequences of lack of randomization in epidemiology/ observation studies

3 Validation of measurement instruments

4 Assumption of normal distribution

5 Subgroup analyses

6 Multiple comparisons

7 Dropouts (intention-to-treat and other fixes)

8 Generalization

\section{Issues and Solutions in Statistical Evaluation of Pharmacological Studies}

Although the above ideal approach 'works', many issues arose over the last 50 years because investigators (1) failed to apply rigorous methods (e.g., those in table 1), (2) sought more conclusions from trials (sometimes termed data dredging) than simply answering the questions posed by the initial hypotheses, or (3) floundered on unresolved ambiguities (table 7).

In 1966, Schor and Karten [16] addressed the issue of what constitutes statistical significance. As part of their statistical evaluation of published clinical trials, then in a generally unsatisfactory state, they proposed a standard of $\mathrm{p}<0.05$ in randomized trials (e.g., to accept falsification of the null hypothesis). The smaller the initial study $\mathrm{p}$ value, the more likely a replicated trial would repeat $p<0.05$. For licensure of new drugs, the FDA now generally requires replicate (large, often placebo-controlled, blinded where appropriate) controlled clinical trials (both $p<0.05)[1,7]$. Since the chance of obtaining $p<0.05$ in two trials is multiplicative, the chance of obtaining two, replicative trials with $\mathrm{p}<0.05$ is $\ll 0.01$. Although the choice of $\mathrm{p}<0.05$ in clinical trials was arbitrary, it is now commonly used and widely accepted. However, the choice of $\mathrm{p}<0.05$ in certain situations, e.g., validation of questionnaires, is not in our opinion generally reasonable as discussed below.

Bradford Hill [17] (1965) confronted the problem of lack of randomization in epidemiology/observation studies (including retrospective case-control and cross-sectional studies, and prospective cohort studies), particularly those attempting to assess causal relations [11]. Following Hill, Sackett [18] described in detail the numerous types of bias that can occur in nonrandomized trials. Hill [17], Sackett [18], Feinstein [6] and others [5, 19] outlined rigorous criteria for attempting to overcome potential bias and confounding that arose in epidemiology/observation trials from lack of randomization. These criteria included quantitatively important effects, 'dose-response' data, and biological plausibility. Hill [17] believed that statistical analyses of epidemiology/observation trials seeking meaningful associations (such as between drug use and effect) were fraught with danger, since one critical methodological assumption (i.e., randomization) could not be met. Newer methods to avoid confounding (e.g., multivariate analyses) are applied after the fact and may yield inappropriate conclusions since they can also be biased.

Validation of instruments (e.g., questionnaires) is crucial to obtain 'true' research. Instruments should be precise (i.e., have small errors on repeat measurements), accurate (measure the true value), and valid (table 5). Methods to accomplish these objectives are generally available $[6,19]$.

Assumption of a normal distribution and use of standard parametric statistics are sometimes not reasonable approximations to the truth $[2,3]$. For example, treatments that lower mean values often decrease variance in the treated groups substantially (heteroscedasticity) [14]. Thus, in these cases, statistical analyses based on the assumption of a normal distribution and homogeneity of variance are inappropriate. Corrections must be made for this effect. If there is uncertainty, robust nonparametric statistics will often solve the problem $[3,14]$.

Investigators frequently wish to determine if subgroups in controlled trials are affected by, or benefit from, a particular treatment. Unless these comparisons are few and specified in advance, they must be considered exploratory. At $\mathrm{p}<0.05$, approximately one in twenty replications (assuming no treatment effect) may be positive by chance alone. Thus, in a controlled trial with a positive primary hypothesis, if multiple subgroup comparisons are made, a statistical correction should be applied [14].

The problem of dropouts or missing data from studies can be serious. If too many subjects or patients drop out completely (disappear) or refuse to participate, then data are incomplete and difficult to evaluate accurately. In controlled trials, intention-to-treat analyses attempt to overcome patient loss and noncompliance [5]. Patients are placed in the initial randomization category. If an outcome is incontrovertible (e.g., death) and observable, even if the patient did not comply with the therapy, then if the study is positive (e.g., drug better than placebo; $p<$ $0.05)$, this suggests that the treatment is truly effective. With too many dropouts, however, no clear believable solution exists for the dropout problem. 
Table 8. Misuse or overinterpretation of statistical analyses

1 Clinical versus statistical significance

2 Nonrandomized studies

(a) Fundamental flaw

(b) Fixes

3 Use of incomplete data sets in meta-analysis

4 Adverse experience reporting

5 Genetic association studies

(a) Microarray analyses

6 Missing data in trials

7 Inadequate validation of critical variables

8 Subpopulation (subgroup) analyses

Issues of generalization from controlled trials to entire populations or to an individual in the population include complex problems in logical probability beyond the scope of this commentary [12]. However, if the sample selected is representative of a carefully defined population, generalization from the trial to the population or individual in the population is more likely to be correct.

\section{Current Tribulations in Pharmacology and Statistics}

In recent publications, we and others have described notable successes in pharmacological sciences over the last decades $[1,4,7,9,10]$. Examples include development of the ACE inhibitors, statins, proton pump inhibitors, and highly effective vaccines (e.g., the hepatitis B vaccine which also prevents cancer of the liver) $[1,7,8]$. These drugs and vaccines have tangibly decreased morbidity/ mortality and brought enormous benefit to patients. Many are now available generically. Clinical trials establishing the value of these treatments and the attendant statistical analyses are exemplary [1, 7-9].

Despite these successes, large numbers of vocal critics of pharmacological sciences and the pharmaceutical industry suggest there is too much disease mongering; stilted, biased framing of questions and hypothesis testing; bias in data collection and analysis; jumping to conclusions unjustified by the data and selectively publishing only 'favorable' data [20]. Moreover, there is extensive 'circular epidemiology', i.e., endlessly repeating the same experiment, sometimes with the same incorrect result [21]. These general criticisms have merit; in table 8 , we summarize certain issues clearly related to statistical analyses of pharmacological studies.

For decades, the notion has been emphasized that statistical significance does not necessarily translate into clinical significances. In 2002, we analyzed several widely touted drugs with statistically significant, but not clinically important benefits [22]. Examples included tolteradine for bladder urgency/incontinence, tacrine for Alzheimer's disease and loratadine $(10 \mathrm{mg})$ for allergic rhinitis [22]. We reviewed not just published data, but unpublished data obtained from the FDA under the Freedom of Information Act (FOI) [22]. As noted above, for registration of drugs, the FDA in the past generally required two large replicate trials (with $\mathrm{p}<0.05$ ), but has not demanded demonstration of 'clinical significance'. (Moreover, as noted in the FOI for $10 \mathrm{mg}$ loratadine, one half of loratadine versus placebo trials were in fact not positive; $p>0.05[22]$.)

A more recent example of a drug with statistically significant findings only in some studies, but without clinical significance (as defined previously by us) is montelukast for allergic rhinitis [22-24]. In the FDA-approved montelukast label for allergic rhinitis, the mean benefit of montelukast and a comparator, loratadine $(10 \mathrm{mg})$ (net of placebo), for the primary endpoint (nasal symptom score) was 7 and 14\%, respectively [23]. Thus, montelukast is numerically less effective than loratidine which we and others consider marginally effective at best for allergic rhinitis [22]. However, an 'objective' reviewer (the Medical Letter on Drugs and Therapeutics) surprisingly failed to point out the minimal quantitative benefit of montelukast for allergic rhinitis [24]. In their evaluation, the Medical Letter on Drugs and Therapeutics discussed the presence or absence of statistical significance in several studies, but not the minimal clinical benefit described in the company label [24]. In the end, patients and physicians want to know the magnitude of the benefit or lack thereof, not the statistical significance of individual studies.

Nonrandomized studies claiming or implying causal associations continue to be performed and published [8, $10,11]$, even when they do not satisfy standards for credible science described by Hill [17], Schor and Karten [16], Sackett [18], and Feinstein [6]. Such studies suffer from the inescapable fundamental flaw of nonrandomization. Moreover, attempts to avoid confounding after the fact are often guesswork and inadequate. Recent examples of flawed epidemiology/observation studies include the use of hormone replacement therapy (HRT), megavitamin E, reduction of blood homocysteine with vitamins, and low fat diets, all of which were incorrectly claimed to reduce cardiovascular risk based on nonrandomized studies [8, $10,11]$.

A widely quoted and extremely influential trial (a large, long-term cohort study, the Nurses' Study) exam- 
Table 9. Comparison of Nurses and 4S Studies

\begin{tabular}{lll}
\hline Variable & Nurses Study (1996) [25] & 4S (1994) [26] \\
\hline Primary hypothesis (endpoint) & $\begin{array}{l}\text { less heart attacks and cardiovascular deaths than } \\
\text { no therapy } \\
\text { estrogen/progesterone (HRT) }\end{array}$ & less deaths than placebo \\
Dependent variable & no & simvastatin \\
Randomization & no & yes \\
Blinding & 59,337 & yes \\
Numbers of subjects & no (see text) & 4,444 \\
Validation of instruments & yes; multivariate analyses to minimize confounding & yes \\
Post-hoc fixes & nomplete; unreported number of dropouts & complete \\
Follow-up of subjects & not measured independently & surrogate (lowering of cholesterol) \\
Compliance & HRT decreases heart attacks/death (see text) & $30 \%$ (ptatin decreases all-cause death by \\
Statistical analysis & & results confirmed and extended in \\
& erroneous (causal) association & multiple trials [8] \\
\hline
\end{tabular}

ined the hypothesis that HRT with estrogen/progestin reduced heart attacks and cardiovascular death (table 9) [25]. The authors of this nonrandomized study claimed more than $60 \%$ reduction in relative risk $(\mathrm{RR}=0.39 ; 95 \%$ confidence interval $0.19-0.78$; $\mathrm{p} \ll 0.05$ ) in women who took HRT compared to those who did not. Table 9 contrasts the Nurses' Study with the randomized 4 S study [26], which proved the value of a now generic statin (simvastatin) in decreasing death from any cause. Potential problems with the Nurses' Study are manifold and obvious (table 8). The results of the Nurses' Study (table 9) were subsequently overturned in controlled, randomized trials of primary and secondary prevention $[8,10]$. Not only was HRT not beneficial; it actually increased cardiovascular events. At least a hundred incorrect or inconclusive epidemiology/observation studies on HRT use were published; in the end, they created confusion and exemplify circular epidemiology and the severe consequences of nonrandomized studies $[8,10,11]$.

One notable post-hoc 'fix' in the Nurses' Study (table 9) was the attempted 'correction' of potential confounding of the data by applying a multivariate analysis for saturated fat intake and use of vitamin E [25]. Recent controlled clinical trials indicate that these variables per se exert no effect on heart attacks and death [8]. Accordingly, these post-hoc fixes only further confounded the Nurses' Study.

By contrast, in the $4 \mathrm{~S}$ trial, since the primary endpoint (mortality) had $\mathrm{p} \ll 0.001$ (table 9) and all patients were accounted for as dead or alive at the end of the study, there was a greater than $99 \%$ chance that an exact repeat of the trial would show $\mathrm{p}<0.05$ [26]. For this reason the FDA rapidly accepted the $4 \mathrm{~S}$ study results as conclusive, and the scientific community considered the decades long 'cholesterol controversy' settled $[8,9,26]$.

Meta-analysis provides an important addition to clinical and statistical armamentaria. However, these analyses obviously depend on complete access to all relevant data. If only positive studies are included, results are biased. An example is the meta-analyses of the efficacy of both the tricyclic (TCA) and serotonin reuptake inhibitor (SSRI) antidepressants [27]. Approximately $40-50 \%$ of antidepressant trials (vs. placebo) with TCAs or SSRIs are not positive $(\mathrm{p}>0.05)$; many of these are not published [27]. Hence, meta-analysis of only published antidepressant trials is biased, unfairly favoring efficacy of the drugs. With complete access to and meta-analysis of all trials of certain antidepressants, both TCAs and SSRIs are more effective than placebo, but the patient response differential (efficacy) is small ( $\sim 10 \%)$ [27]. In view of the serious side effects of these drugs, their clinical utility (benefit/risk ratio) is being widely debated.

Efficacy claims in clinical trials with antidepressants and loratadine exemplify an important statistical principle $[22,27]$. When clinical trials of such drugs (i.e., possessing a small drug effect and a fairly large variance) are performed with a resultant $\mathrm{p} \sim 0.05$, then an exact replication of such a trial has approximately a $50 \%$ chance of yielding a $p>0.05$ [11]. This helps to explain why many TCA and SSRI antidepressant trials fail - the drugs barely 'work' on the average. Numerous reviews of these types of drugs (e.g., the antidepressants) in prominent journals claim that these drugs are effective but the extremely modest efficacy (based on a complete analysis of both 
positive and negative clinical trials) is neither quantitatively nor explicitly described [28].

Another difficult issue in clinical trials of drugs is evaluation and reporting of adverse drug experiences (AE). Even more complex is the assessment of AE causality [1]. In large, long-term placebo-controlled trials, hundreds of AEs on drug and placebo are collected. Even if no true AE occurs and, for the sake of argument, $200 \mathrm{AE}$ types are analyzed, approximately ten by chance alone will have $\mathrm{p}<0.05$, unless multiple comparison testing is employed. In our view, although a statistical analysis is helpful, no substitute exists for inspection, evaluation by methods described by Hill [17], ourselves [1], and others, and clinical judgment, e.g., clinical importance, biological plausibility, effect size, etc.. Relying on statistical analyses of AEs alone is problematic. This issue was recently addressed by the FDA since the current labeling system for $\mathrm{AE}$ reporting is grossly unsatisfactory. The problem of AEs that occur with marketed drug use is beyond the scope of this article and even more complex.

Recently, because of tremendous advances in technology, genetic association studies are increasingly performed and reported $[29,30]$. But serious issues arise with execution and interpretation. Many published statistically significant associations between marker genes and genes of interest, such as silent disease genes, could not be replicated and had to be repudiated, leading some journals to question whether such association studies merited publication [31]. Efforts were made to establish uniform criteria to enhance reliability [31]. In association studies involving messenger RNA (e.g., micoarray analysis), two general issues occur. First, there are technical issues, e.g., recovery of RNA from various tissues. [Unless recovery for each individual RNA species is measured with external and internal standards, one cannot be certain that a small difference in screening (e.g., by a factor of 2 or 3 ) is real. This is analogous to the problem in epidemiology/observation studies.] Second, since there may be hundreds, if not thousands of variables, the best statistical techniques to employ in drawing causal conclusions about associations are unclear [29-31]. Accordingly, most current studies should be viewed as hypothesis generating. Moreover, the scientific basis of microarray, proteomic and many phenotypic assays in cancer studies is now being challenged due to heterogeneity of even cloned cells in some cases - the stem cell problem [32].

Missing data (table 8) also pose difficult problems, especially in the primary endpoint. The $4 S$ trial (table 9), in which every patient of the 4,444 was assessed as alive or dead at the termination of the trial, is exemplary but atyp- ical [26]. Some trials have so many noncompliant patients or dropouts that no reliable conclusions can be drawn, since the outcome for noncompliant patients or dropouts may differ greatly from those patients remaining in the trial. A recent example is the $\mathrm{A}-\mathrm{Z}$ trial of simvastatin (vs. placebo) for the therapy of patients with acute coronary syndromes [33]. Approximately $33 \%$ discontinued active therapy rendering definitive conclusions impossible [33].

In publications, criteria for acceptable validation of instruments, e.g., questionnaires, used in clinical trials are unclear. However, Sempos [19] emphasized that semiquantitative questionnaires are often used with the claim that they are validated because a positive association occurs between responses and a 'gold standard' $(\mathrm{p}<0.05)$. However, this is a very loose standard, as disclosed by inspection of the data in such validation studies. As is well known, even very weak associations with correlation coefficients ( $\mathrm{r}$ values) of between 0.2 and 0.4 may have $\mathrm{p}<$ 0.05 if enough subjects are studied. In our view, there is often unacceptable variability with $\mathrm{p}<0.05$ as the sole criterion. Such unreliable instruments contribute to misleading results and beliefs. This is especially unfortunate since powerful methods for questionnaire validation are available [34]. For example, even in asthma, a disease with notoriously difficult-to-measure subjective symptoms, it has been possible to design validated symptom questionnaires, as well as measure minimally perceivable improvement [34].

Another thorny problem involves drug effect on subgroups in trials. In general, a primary hypothesis is posed and perhaps one or two secondary hypotheses. The primary hypothesis usually deals with the mean or median, or with a life table analysis, but reveals nothing about subgroups. In our view, if the primary hypothesis is substantiated, it is reasonable to investigate subgroups by age, sex, severity of disease, etc. but these should be considered only exploratory. In a recent highly controversial analysis of the double-blind, placebo-controlled, 15,603-patient CHARISMA trial (Clopidogrel and Aspirin versus Aspirin alone for the Prevention of Atherothrombotic Events), the primary endpoint (the composite of myocardial infarction, stroke or cardiovascular death) was negative (relative risk $0.93 ; \mathrm{p}=0.22)[35,36]$. The authors, however, 'suggested' that one subgroup might benefit from the combination compared to aspirin alone, whereas another subgroup would be harmed [35, 36]. Two editorialists criticized this analysis, in our opinion, correctly $[35,36]$. Since the trial was negative, subgroup analysis added little.

In summary, we have outlined published instances of misuse or abuse of the sound and productive statistical 
and clinical trial principles described by Schor and Karten [16], Hill [17], Sackett [18], Feinstein [6] and many others [5], recently reviewed by Reidenberg [4] and ourselves [1, 7-11]. Sometimes these problems arise from lack of understanding but in other cases, from faulty publication standards. Fundamental changes are needed to assure higher standards are observed in research and publications.

\section{Recommendations}

In accordance with the scientific bases of pharmacology, including proper, productive statistical principles and practices described above, we propose a series of recommendations to improve the probability of obtaining and publishing reproducible, correct results.

The following recommendations are suggested. To improve the situation immediately, we suggest that journals provide explicit guidance to reviewers and editors for various types of studies. At present, journals generally send submissions to outside reviewers who are considered 'experts' [37]. This is termed the 'peer review' process. Little guidance, however, is offered to reviewers, except notions like creativity, originality, importance, appropriate methods and the like [37]. We submit that such minimal guidance sometimes encourages publication of poorly designed and executed studies. Current practices do not discourage circular epidemiology and other wasteful practices discussed above. Stricter criteria for publication will decrease erroneous publications. We suggest the following:

1 In both controlled basic (including animal) and clinical studies, the principles outlined in tables 1 and 8 (4S study) should be followed. We urge that all animal and clinical experimentation, where appropriate, be randomized, blinded, and properly controlled after a hypothesis and sample size needs are formulated. (Bias often creeps into nonrandomized, unblinded animal as well as clinical studies.) No sound reason exists to avoid doing this and the results then become more persuasive. Also, studies with too many dropouts (no matter how expensive) are often uninterpretable and unworthy of publication or belief.

2 Authors and reviewers should comment on the clinical or pharmacological significance of results. To claim that a drug is better than placebo or control according to the Schor criterion (mean or median value, $p<0.05)$ reveals nothing about the quantitative benefit or pharmacological effect. With large numbers of subjects, very small, meaningless average differences can be detected with $p<0.05$. Moreover, the benefit or size of effect should be put into context and, where possible, compared with positive controls (e.g., other therapy).

3 In epidemiology/observation studies, we believe that the Hill criteria must be satisfied in order to even implicitly suggest causal associations [11-17]. Associations with relative risks or odds ratios of less than 2 are fraught with difficulty. Kuller [21] and Smith [38] eloquently addressed these issues. We must avoid 'circular epidemiology' where tens or hundreds of studies are performed (e.g., on cholesterol or HRT epidemiology), all of which are inconclusive or incorrect $[21,38]$. To answer certain efficacy or safety questions, one must perform randomized, controlled experimental trials. In our view, a relative risk or odds ratio below 2 in an epidemiology/observation study requires substantial justification to be published.

4 More guidance must be provided for genetic association studies to avoid false, misleading associations. We recommend tentatively the following: for example, in mRNA microassay studies, the authors should prove that specific RNAs are indeed truly elevated (or decreased) beyond chance with proper controls, recovery experiments, etc. This can be achieved with quantitative techniques now available. To assure real association and publication, we believe investigators should then perform an additional step. In this second part of the experiment, investigators should hypothesize changes in one or a few specific genes of interest and, in a new sample, test the hypothesis. The statistical issues in the second step are straightforward. They should also test whether the proteins of interest are also elevated, the ultimate step. If confirmed, results could then be published but as noted above, conclusions must be tempered by assessing biological significance [32].

5 To be meaningful a meta-analysis must access all the data, not just positive published trials. If this objective cannot be achieved (i.e., if negative studies are not published or available), the meta-analysis can only provide an upper limit on efficacy or effectiveness [1]. This serious limitation should be noted and emphasized.

6 Validation of methods is mandatory [39]. In the Nurses' Study (table 8) [25], determination of estrogen use (the key variable) was assessed with a nonvalidated questionnaire. We believe such practices are unacceptable. Journals should insist on appropriate valida- 
tion for the hypothesis testing at hand, as done with the asthma questionnaires [34].

7 Subgroup analyses (post-hoc) must be viewed with extreme caution even in positive studies, unless the $\mathrm{p}$ values are highly significant (after correction for multiple comparisons) [39].

\section{Conclusions}

The pharmacological and statistical methods described above, when rigorously applied, permit proper testing of hypotheses in both basic and human pharma- cology $[8,9,39]$. Although this process is often expensive and time consuming, the outcome will be fewer falsepositive and false-negative pharmacological studies, eventually saving much time and money, and most importantly, correct data and information to help patients and their health care providers.

\section{Acknowledgements}

The authors wish to thank Michiko Spector for help in the preparation of the manuscript and Dr. George Williams, Dr. Beth Seidenberg and Dr. June Spector for their review of the manuscript.

\section{References}

1 Spector R: The Scientific Basis of Clinical Pharmacology: Principles and Examples. Boston, Little, Brown, 1986.

2 Murphy EA: A Companion to Medical Statistics. Baltimore, Johns Hopkins University Press, 1985

3 Bradlee JV: Distribution-Free Statistical Tests. Englewood Cliffs, Prentice-Hall, 1968.

4 Reidenberg MM: Clinical pharmacology: the scientific basis of therapeutics. Pharmacologist 1999;41:100-106.

5 Jenicek M: Foundations of Evidence-Based Medicine. Boca Raton, Parthenon, 2003.

6 Feinstein AR: Clinical Biostatistics. Saint Louis, Mosby, 1977.

7 Spector R, Vesell ES: The power of pharmacological sciences: the example of proton pump inhibitors. Pharmacology 2006;76: 148-156.

8 Spector R, Vesell ES: The heart of drug discovery and development: rational target selection. Pharmacology 2006;77:85-92.

-9 Spector R: Progress in the search for ideal drugs. Pharmacology 2002;64:1-7.

10 Spector R, Vesell ES: Which studies of therapy merit credence? Vitamin $\mathrm{E}$ and estrogen as cautionary examples. J Clin Pharmacol 2002;42:955-962.

11 Spector R, Vesell ES: The pursuit of truth: role of epidemiology/observation studies. Clin Pharmacol 2000;40:1205-1210.

12 Carnap R: Introduction to the Philosophy of Science. New York, Dover, 1995.

13 Popper KR: Objective Knowledge. Oxford, Clarendon Press, 1973.

14 Colquhoun D: Lecture on Biostatistics. Oxford, Clarendon Press, 1971.
15 Hill AB: Principles of Medical Statistics, ed 9. New York, Oxford University Press, 1971.

-16 Schor S, Karten I: Statistical evaluation of medical journal manuscripts. JAMA 1966; 195:145-150.

17 Hill AB: The environment and disease: association or causation? Proc R Soc Med 1965; 58:293-300.

18 Sackett DL: Bias in analytic research. J Chronic Dis 1979;32:51-63.

19 Sempos CT: Some limitations of semiquantitative food frequency questionnaires. Am J Epidemiol 1992;135:1127-1132.

20 Levi R: Science is for sale. Skeptical Inquirer 2006;30:44-46.

21 Kuller LH: Invited commentary: circular epidemiology. Am J Epidemiol 1999;150:897903.

22 Spector R, Vesell ES: A rational approach to the selection of drugs for clinical practice. Pharmacology 2002;65:57-61.

23 Singulair $^{\circledR}$ (Montelukast Sodium) Tablets, Product Description. Merck, 2002.

24 Montelukast (Singulair ${ }^{\circledR}$ ) for perennial allergic rhinitis. Med Lett Drugs Ther 2005;47: 87-88.

25 Godstein F, Stampfer MJ, Manson J, et al: Post menopausal estrogen and progestin use and the risk of cardiovascular disease. $\mathrm{N}$ Engl J Med 1996;335:453-461.

26 Scandinavian Simvistatin Survival Study Group: Randomized trial of cholesterol lowering in 4,444 patients with coronary heart disease: The Scandinavian Simvistatin Survival Study (4S). Lancet 1994;344:13831389.

27 Moncrieff J, Kirsch I: Efficacy of antidepressants in adults. BMJ 2005;331:155-157.

28 Mann JJ: The medical management of depression. N Engl J Med 2005;353:18191834.
29 Quackenbush J: Microarray analysis and tumor classification. N Engl J Med 2006;354: 2463-2472.

30 Harris NL, Horning SJ: Burkitts' lymphoma - the message from microarrays. N Engl J Med 2006;354:2495-2497.

31 Page GP, George V, Go RC, Page PZ, Allison DB: 'Are we there yet?': deciding when one has demonstrated specific genetic causation in complex diseases and quantitative traits. Am J Hum Genet 2003;73:711-719.

32 Dean M: Cancer stem cells. Mol Interv 2006; 6:141-147.

33 De Lemos JA, Blazing MA, Wiviott SD, et al: Early intensive vs. a delayed conservative simvistatin strategy in patients with acute coronary syndromes. JAMA 2004;292:13071316.

34 Santanello NC, Zhang J, Seidenberg B, Reiss T, Baber BL: What are minimal important changes for asthma measures in a clinical trial? Eur Respir J 1999;14:23-27.

35 Lagakos SW: The challenge of subgroup analyses - reporting without distorting. N Engl J Med 2006;354:1667-1669.

36 Pfeffer MA, Jarcho JA: The charisma of subgroups and the subgroups of charisma. $\mathrm{N}$ Engl J Med 2006;354:1744-1746.

37 Reidenberg JW: Improving peer review: a guide for reviewers of biomedical research. Clin Pharmacol Ther 2002;72:469-473.

38 Smith GD: Reflections on the limitations of epidemiology. J Clin Epidemiol 2001;54: 325-331.

39 Guidance for Industry: E9 Statistical Principles for Clinical Trials: US Department of Health and Human Services, Food and Drug Administration, September 1998. 